\section{PSQ-042 MODIFICATION ON FASTING LIPID AND RENAL PARAMETERS IN PATIENTS SWITCHING FROM TENOFOVIR DISOPROXIL TO TENOFOVIR ALAFENAMIDE}

1P Taberner Bonastre, ${ }^{2} \mathrm{~L}$ Vallez Valero*, ${ }^{2} \mathrm{SM}$ Cano Marrón, ${ }^{3} \mathrm{~T}$ Puig Ganau, ${ }^{2} \mathrm{~B}$ Amoros Folguera, ${ }^{1} \mathrm{Fl}$ Torres Bondia, ${ }^{2} \mathrm{JA}$ Schoenenberger Arnaiz. ${ }^{1}$ Hospital Universitario Santa Maria, Hospital Pharmacy, Lleida, Spain; ${ }^{2}$ Hospital Universitario Arnau De Vilanova De Lleida, Pharmacy, Lleida, Spain; ${ }^{3}$ Hospital Universitario Arnau De Vilanova De Lleida, Infectious Diseases, Lleida, Spain

\subsection{6/ejhpharm-2019-eahpconf.475}

Background Tenofovir alafenamide (TAF) in clinical trials demonstrated less impact than tenofovir disoproxil (TDF) in affecting renal and bone parameters, whereas TDF protects from hypercholesterolaemia and hypertriglyceridaemia.

Purpose To analyse in clinical practice of human immunodeficiency virus-infected (HIV-infected), how renal function and fasting lipid parameters are modified when switching TDF to TAF. As a second aim, to evaluate effectiveness and the immunological system.

Material and methods Retrospective observational study (July 2016 to August 2018) conducted in HIV-infected patients treated for $\geq 6$ months with a TDF regimen who switched to a TAF regimen kept $>48$ weeks. We considered virological success if HIV-1 RNA $<35$ copies/mL.

Demographic variables were registered. Follow-up variables: serum-creatinine, phosphataemia, glomerular filtration rate (GFR calculated by CKD-EPI), total cholesterol (TC), hightdensity-lipoprotein (HDL), low-density-lipoprotein (LDL), triglycerides, CD4 + cell counts and HIV RNA-concentration.

Two-sided $t$-student test was used for comparing pre-post variables except for GFR with two-sided Wilcoxon signed-rank test. We used Pearson correlation coefficient ( $r$ ) evaluating the relation with TC and HDL-LDL.

Variables were extracted from: electronic clinical records (SAP) and the pharmacy-dispensation program (Silicon). The statistical data were analysed with SPSS.

Results Forty-eight patients were included, mean age 44 years (range 21-70), 79.2\% males. Most received antiretroviral treatment (ART) with emtricitabine/elvitegravir/cobicistat (44/48).

There were significant differences from baseline to 48 weeks with serum-creatinine, TC, HDL and CD4+. Serumcreatinine decreased $0.08 \mathrm{mg} / \mathrm{dL}$ with TAF $(0.98 \pm 0.18 \mathrm{mg} / \mathrm{dL}$ with TDF, $\mathrm{p}=0.0001)$; TC, HDL and CD4 were greater with TAF; difference $19.8 \mathrm{mg} / \mathrm{dL} \quad(173.4 \mathrm{mg} / \mathrm{dL}$ with TDF, $\mathrm{p}=0.0001), 8.7 \mathrm{mg} / \mathrm{dL}(47.6 \mathrm{mg} / \mathrm{dL}$ with TDF, $\mathrm{p}=0.0001)$ and 76 cells $/ \mu \mathrm{L}$ (694.2 cells/ $\mu \mathrm{L}$ with TDF, $\mathrm{p}=0.02)$ respectively. There were no significant differences with phosphataemia, LDL and TG, but all increased with TAF (difference 0.06, 8.03 and $10.77 \mathrm{mg} / \mathrm{dL}$, concentration with TDF 3.31, 106 and $115.4 \mathrm{mg} / \mathrm{dL}$ respectively; $\mathrm{p}>0.05)$. There were no statistical differences with GFR ( $p>0.05)$.

Cholesterol correlated with LDL $(p=0.0001 ; r=0.94)$, but not with HDL ( $p>0.05 ; \mathrm{r}=0.03)$.

All patients achieved virological success, even three patients with RNA-concentration $>35$ copies/mL before switching.

Conclusion After 48 weeks of patients, in clinical practice, who changed to TAF on their ART, $100 \%$ of patients archived virological suppression, with reduction in serum-creatinine and improvement in the immunological system. Nevertheless, hypercholesterolaemia was observed based mainly on LDL elevation.

\section{REFERENCES AND/OR ACKNOWLEDGEMENTS}

No conflict of interest.

\section{PSQ-043 COMORBIDITIES, POLYPHARMACY AND ADHERENCE IN GERIATRIC HIV PATIENTS}

JM Vinuesa Hernando*, M Gimeno Gracia, T Salvador Gómez, MDP Pardo Jario, MA Alcacera, S Gamarra Calvo, FJ Campos Montellano, B Bonaga Serrano, AJ Frutos PerezSurio. Hospital Clínico Universitario Lozano Blesa, Pharmacy, Zaragoza, Spain

\subsection{6/ejhpharm-2019-eahpconf.476}

Background HIV patients live longer, and as a result are more exposed to comorbidities and even earlier onset. This leads to a polypharmacy situation, with the consequent risk of adverse effects, interactions and lack of adherence.

Purpose To describe the prevalence of comorbidities, polypharmacy and adherence in the HIV population with antiretroviral treatment (ART) over 65 years of age.

Material and methods Retrospective observational study of the HIV population with ART of a third-level hospital, which between January and July of 2018 had an age of $\geq 65$ years. Polypharmacy was defined as the use of six active ingredients (AI) or more, high polypharmacy using more than 11 and extreme polypharmacy using more than 21 AI (including ART). Demographic, clinical and pharmacotherapeutic characteristics were studied. Patients who took at least $90 \%$ of their prescribed ART were classified as good adherers. The comorbidities recorded were hypertension (HT), diabetes mellitus (DM), dyslipidaemia (DSP) and central nervous system (CNS) disorders. The sources of information used were electronic prescribing, clinical history and personal interviews.

Results The patients that met inclusion criteria were 36, of which $77 \%$ were males and the mean age was 72.19 years (SD 5.69). Mean age at diagnosis was 57.08 years (SD 9.51). Five patients died during the study period and one did not want to participate, so 30 patients took part in the subsequent analysis. Regarding comorbidities: $56.7 \%$ of the patients suffered from HT, 26.7\% from DM, 70\% from DSP and $43.3 \%$ from any CNS-related pathology. Then, of the 30 patients interviewed, 90\% presented with polypharmacy and50\% of those, a high polypharmacy situation. The average of concomitant AI was 7.4, however the average AI of the ART was 3.3. Finally, 86\% were adherent patients.

Conclusion The most prevalent comorbidity in this population was dyslipidaemia, followed by hypertension, from which can be deduced the greater cardiovascular risk they face. The polypharmacy of these patients can be explained through the concomitant drugs, because nowadays ART has been simplified. Despite this high degree of polypharmacy, adherence to ART is very good.

\section{REFERENCES AND/OR ACKNOWLEDGEMENTS}

http://dx.doi.org/10.1136/ejhpharm-2013-000436.31

No conflict of interest.

\section{PSQ-044 ASEPTIC MENINGITIS INDUCED BY INTRAVENOUS IMMUNOGLOBULIN}

M Domínguez Bachiller*, MI Barcia Martin, AM Martin de Rosales, M Pérez Encinas. Hospital Universitario Fundación Alcorcon, Hospital Pharmacy, Alcorcon, Spain

\subsection{6/ejhpharm-2019-eahpconf.477}

Background The use of intravenous immunoglobulin (IVIg) for the treatment of different pathologies is increasing and has shown a good safety profile. However, rare but serious 
adverse reactions (AR) such as aseptic meningitis (AM) are described in the product information (PI).

Purpose To describe and analyse five cases of AM in patients treated with IVIg in our centre.

Material and methods A literature search was conducted on the AR of IVIg. The case analysis was established using the Karch-Lasagna algorithm.

Results There were five cases notified of AM in a 3 month period (80\% females). Clinical manifestations included headache, fever, nausea and vomiting, and in some cases photophobia. Symptoms usually commenced within 48 hours after infusion. In all cases lumbar puncture was compatible with AM. Two patients had to be hospitalised due to AM, one of them prolonged hospitalisation.

All patients received IVIg of the same brand, presentation and even some of the same batch. All of them received an individualised administration form prepared by the pharmacist including premedication information and the rate of administration of the IVIg calculated according to patient weight and PI.

The Karch-Lasagna algorithm in these cases established a possible causal relationship between IVIg and the occurrence of AM.

Every case reported had a neurological-based pathology: myasthaenia gravis, nystagmus, multiple mononeuropathy, Parsonage-Turner syndrome and sensitive-motor polyneuropathy. Nevertheless, in our centre the other five patients with no neurological pathology received the same presentation and batch of IVIg during the same period and did not present AM. The analysis leads us to suspect that patients with basic neurological diagnosis have a higher risk of suffering from AM.

The preventive measures adopted were to reduce the speed of individualised administration and to insist that good hydration is important in preventing this adverse effect.

Conclusion IVIg have demonstrated efficacy and a good safety profile in clinical trials. However, possible AR due to its use can be observed. The role of the pharmacist is important in the individualised information by patients concerning the administration of immunoglobulins. In order to reduce the incidence of $\mathrm{AM}$, it is suggested to start the initial infusion at a slow rate, prehydration and premedication therapy.

\section{REFERENCES AND/OR ACKNOWLEDGEMENTS}

No conflict of interest.

\section{PSQ-045 SAFETY PROFILE OF SUNITINIB IN REAL CLINICAL PRACTICE}

${ }^{1}$ A Alcala Soto*, ${ }^{1} \mathrm{C}$ Puivecino Moreno, ${ }^{1} \mathrm{R}$ Gazquez Perez, ${ }^{1} \mathrm{~A}$ Varas Perez, ${ }^{1} \mathrm{~V}$ SanchezMatamoros Piazza, ${ }^{2} \mathrm{~L}$ Jimenez Pichardo, ${ }^{1} \mathrm{~V}$ Vazquez Vela, ${ }^{1} \mathrm{JF}$ Sierra Sanchez, ${ }^{1} \mathrm{R}$ Gavira Moreno, ${ }^{1} \mathrm{MT}$ Gomez de Travecedo Y Calvo. 'Hospital Universitario Jerez de la Frontera, Pharmacy Service, Jerez de la Frontera-Cádiz, Spain; ${ }^{2}$ Hospital San Juan Grande, Pharmacy Service, Jerez De La Frontera-Cádiz, Spain

10.1136/ejhpharm-2019-eahpconf.478

Background In long-term safety studies of sunitinib, most adverse events (AE) occurred initially between the first 6 months and 1 year, and remained stable or decreased in frequency over time.

Purpose To analyse the safety and tolerability of sunitinib in real clinical practice.
Material and methods Retrospective descriptive and observational analysis. All patients treated with sunitinib from April 2010 to September 2018 were selected. Variables collected were: sex, age, diagnosis, line of treatment, date of beginning and end of treatment with sunitinib, reasons for suspension, dose reductions and $\mathrm{AE}$. To assess safety, frequency of adverse reactions, median time to treatment suspension due to $\mathrm{AE}$, median time to dose reductions and the reasons were taken into account. Data was collected from the electronic medical record (DIRAYA) and the prescription program (FARMIS and PRISMA).

Results Thirty-five patients were included, 66\% males, with an average age of 62 years. Eighty per cent of patients $(n=28)$ had metastatic renal cell cancer (mRCC), 11\% $(n=4)$ gastrointestinal stromal tumour (GIST) and $3 \% \quad(n=1)$ pancreatic tumor, unknown $n=2$. Seventy-seven per cent (27) of patients received sunitib as first-line therapy, 20\% (seven) received it as second-line and 3\% (one) as third-line. Most frequent $\mathrm{AE}$ were asthaenia (21 patients), hypertension blood pressure (HBP) (12 patients), mucositis (nine patients), anaemia (eight patients), bleeding and plantar-palmar-syndrome (six patients respectively). Ten patients discontinued treatment due to $\mathrm{AE}$, median time to treatment suspension due to $\mathrm{AE}$ was 3.42 months (0.47-95.43) because of poor tolerance, unacceptable toxicity, haemorrhages, osteonecrosis of the jaw, asthaenia, mucositis, anorexia and liver toxicity. Of these patients, only three had previous dose reductions. Eight patients required dose reduction, with a median time to dose reduction of 1.78 months (0.97-87.37). The main cause of reduction was asthaenia (5/8). One patient had a second dose reduction 1 month after the first reduction due to poor quality of life.

Conclusion Reported AE were within the expected range, with asthaenia and hypertension as the most frequent. About onethird of patients discontinued treatment with sunitinib due to $\mathrm{AE}$ in the first 4 months of treatment and in most cases without prior dose reductions.

\section{REFERENCES AND/OR ACKNOWLEDGEMENTS}

https://www.ema.europa.eu/documents/product-information/ sutent-epar-product-information_en.pdf

No conflict of interest.

\section{PSQ-046 EFFICACY AND SAFETY OF PANITUMUMAB IN METASTATIC COLORECTAL CANCER TREATMENT}

MS Aldirra-Taha*, A Planas-Giner, MÁ Pérez-Quirós, R Rodriguez Mauriz, N Almendros Abad, L Borras Trias, C Segui Solanes, N Rudi Sola. Hospital General de Granollers, Hospital Pharmacy, Barcelona, Spain

\subsection{6/ejhpharm-2019-eahpconf.479}

Background The use of panitumumab in the treatment of metastatic colorectal cancer (mCRC) remains controversial because of its risk/benefit profile.

Purpose The aim of this study was to investigate the efficacy and safety of panitumumab in patients with wild-type KRAS gene in the treatment of mCRC.

Material and methods For this retrospective and observational study, patients diagnosed with mCRC treated with panitumumab monotherapy and in combination with chemotherapy during the period from January 2009 to March 2017 were selected.

Only patients treated with panitumumab for a period longer than 12 weeks were included in the study. 